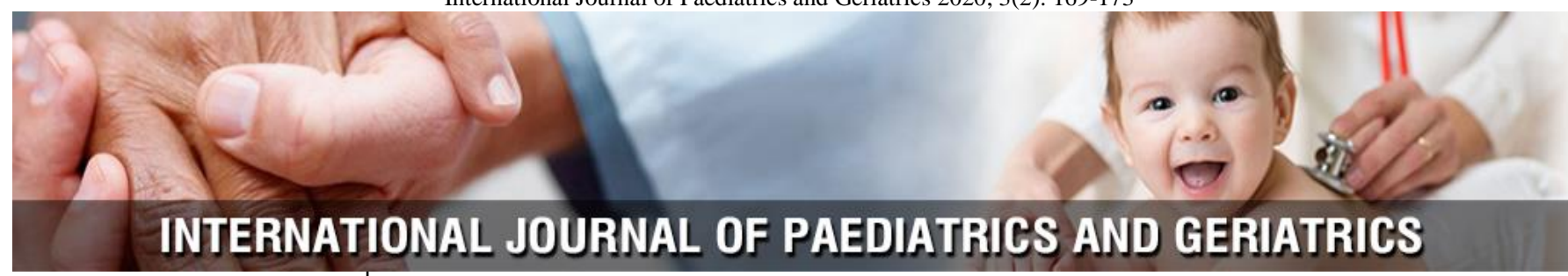

P-ISSN: 2664-3685

E-ISSN: $2664-3693$

IJPG 2020; 3(2): 169-173

Received: 21-08-2020

Accepted: 26-09-2020

Dr. Anand S Ranagol

MD Pediatrics, Assistant

Professor, Department of

Pediatrics, Al Ameen Medical

College and Hospital,

Bijaypur, Karnataka, India

Dr. Savita Koregol

MD DVL, Associate Professor,

Department of Skin and

Veneral Diseases, Al Ameen

Medical College and Hospital,

Bijaypur, Karnataka, India

Dr. Chandrashekhar Bhuyyar

MD Forensic Medicine and

Toxicology, Associate

Professor, Department of

Forensic Medicine and

Toxicology, Al Ameen Medica

College, Bijaypur, Karnataka,

India
Corresponding Author:

Dr. Savita Koregol

MD DVL, Associate Professor,

Department of Skin and

Veneral Diseases, Al Ameen

Medical College and Hospital,

Bijaypur, Karnataka, India

\section{An insight into the childhood obesity and importance of lifestyle counselling on obesity status}

\author{
Dr. Anand S Ranagol, Dr. Savita Koregol and Dr. Chandrashekhar \\ Bhuyyar
}

DOI: $\underline{\text { https://doi.org/10.33545/26643685.2020.v3.i2c.113 }}$

\begin{abstract}
Background: Obese children also suffer from emotional, mental, and social trauma. Obese children are seen to have depression and very low self-esteem. Also, such children are teased by society and have high peer pressure. Additionally, obesity and overweight pose a high burden on the health care system.

Aims: The present trial was carried out to document the efficacy of counselling about physical activity, nutrition, and weight on obese children of low socioeconomic status.

Materials and Methods: The study was carried out on 48 children and was based on the questionnaire on the lifestyle modification and counselling sessions with questions based on healthy habits and physical exercise. Associated comorbidities such as asthma or diabetes were also asked along with their treating paediatrician. The collected data were subjected to statistical evaluation and the results were formulated.

Results: Counselling for nutrition, physical activity, and weight were provided to study participants and the results showed that $80 \%(n=12)$ obese, $66.6 \%(n=6)$ overweight, and $58.3 \%(n=14)$ healthy subjects received counselling for nutrition. In male participants $61.53 \%(\mathrm{n}=16), 69.23 \%(\mathrm{n}=18)$, and $65.38 \%(\mathrm{n}=17)$ respectively were counselled for nutrition, physical activity, and weight. Similarly, in females $68.18 \%(n=15), 63.63 \%(n=14)$, and $63.63 \%(n=14)$ respectively were counselled for nutrition, physical activity, and weight. Regarding diabetics in the study $(n=2)$, all the subjects were counselled for weight, nutrition, as well as physical activity. In subjects with asthma, the counselling for nutrition, physical activity, and weight was given to $63.6 \%(\mathrm{n}=7), 54.5 \%(\mathrm{n}=6)$, and $54.5 \%(\mathrm{n}=6)$ respectively.

Conclusion: The present study concludes that overweight and obese children do not get more counselling sessions compared to their healthy peers. There is a need for more interactions at home and more reinforcement of counselling sessions in the school curriculum with special emphasis on overweight and obese children regarding their nutrition, physical activity, and weight.
\end{abstract}

Keywords: Obesity, overweight, BMI, counselling, childhood obesity

\section{Introduction}

Obesity in childhood is an issue of concern and is increasing worldwide with the recorded increase by triple value since the late $80 \mathrm{~s}$ in the U.S alone. Approximately one-third of children in the age range of 2 years to 19 years are obese and overweight in the United States, despite the NHANES survey showing a plateau in obesity increase in the past decade ${ }^{[1]}$. Obesity has also been linked to ethnicity and socioeconomic status where it is found that obesity is higher in people with low socioeconomic status compared to high. Increasing obesity in children is an issue of concern as obesity and overweight is linked to associated morbidities and complications. Various complications being linked to obesity are diabetes, hypertension, sleep apnea, liver diseases, dyslipidaemia, and insulin resistance ${ }^{[2]}$. Obesity acquired during childhood tends to remain till adult life leading to early life mortality and morbidity. As obesity and hence mortality, and morbidity are increasing in the present generation, hence, there might be a decrease in life expectancy in the present generation owing to this obesity ${ }^{[3]}$.

Obese children also suffer from emotional, mental, and social trauma. Obese children are seen to have depression and very low self-esteem. Also, such children are teased by society and have high peer pressure. Additionally, obesity and overweight pose a high burden on the health care system ${ }^{[4]}$. As obesity remains with an individual till adulthood, it also poses long-term financial constrain on the economy. It is estimated that in the future this obesity in children will increase highly. 
Hence, its prevention and insight into this problem are necessary. Till data the data regarding epidemic and strategies for diagnosing, prevention, and control is limited [5].

Cochrane review of 2011 suggested various measures for controlling obesity and overweight in childhood such as implementing physical activity in schooling schedule, focus on healthy lifestyle in children, healthy diet, parent's involvement in outdoor and physical activities, and improving the home eating habits ${ }^{[6]}$. These studies included in the Cochrane review failed to develop generalized recommendations in the prevention and management of obesity owing to the differences in the various included studies. In the recent work done in literature, it is concluded that lifestyle modification, behavioural modifications, and diet modification are highly effective in successfully reducing obesity and adiposity with positive alterations in BMI. However, the most effective therapy was not suggested by any study ${ }^{[7]}$.

Orlistat, a pharmacologic agent has been tried in treating obesity and is found to be an effective agent for reducing obesity. However, Orlistat is found to have severe life effects worse than obesity itself. Despite various interventions under investigation as effective measures for treating and managing obesity, there is no therapy still considered as standard for treating obesity ${ }^{[8]}$.

It is shown that among children, who have obesity, only a few of them are diagnosed with obesity. Hence, only those who are diagnosed get the information on reducing obesity in terms of diet counselling, physical activity, and behavioural modifications. Hence the present trial was carried out to evaluate the efficacy of implementing and identifying a novel strategy for preventing obesity in childhood. Also, the study was aimed at documenting the efficacy of counselling about physical activity, nutrition, and weight on obese children of low socioeconomic status.

\section{Materials and Methods}

The present trial was carried out to evaluate the efficacy of implementing and identifying a novel strategy for preventing obesity in childhood. Also, the study was aimed at documenting the efficacy of counselling about physical activity, nutrition, and weight on obese children of low socioeconomic status. The trial included 48 children from the age group of 8 years to 14 years with a mean age of 10.12 years. The informed consent for the study was obtained from the parents of the participating children. The Ethical Committee of the institute approved and gave Ethical clearance for the study.

All 48 participants were from the grade 6 t to grade $8^{\text {th }}$ and were selected randomly from 5 participant schools. The inclusion criteria for the study were subjects who could understand the instructions, could understand both English and Hindi, and were overweight and obese. The exclusion criteria of the study were individuals if their parents did not give consent to participate in the study, did not participate in the explanatory session, children who were underweight with BMI of less than $5^{\text {th }}$ percentile as the included subjects were counselled regarding nutrition, weight, and lifestyle modification including physical activity.

All the measurements of the physical dimensions were taken by an experienced in the field. These physical measurements were recorded during the exercise session. The identity of the study subjects was kept confidential and numbers instead of names were used for identification purposes. The height and weight of the study subjects were also recorded.

The study was based on the survey where the survey questionnaire, where questions were read out loud to the students so that participants could understand the questions clearly, and the students entered their responses on the respective computers.

The survey also included questions based on lifestyle modification and counselling sessions with questions based on healthy habits and physical exercise. The BMI for each subject was calculated from the height and weight. Other demographic characteristics taken into account were gender, socioeconomic status, and weight. To define obesity and overweight, BMI which was less than $95^{\text {th }}$ percentile and more than $85^{\text {th }}$ percentile was the marked points. The socioeconomic status was divided into low, medium, or high. Associated comorbidities such as asthma or diabetes were also asked along with their treating paediatrician. The collected data were subjected to statistical evaluation and the results were formulated.

\section{Results}

The present trial was carried out to evaluate the efficacy of implementing and identifying a novel strategy for preventing obesity in childhood. Also, the study was aimed at documenting the efficacy of counselling about physical activity, nutrition, and weight on obese children of low socioeconomic status. The trial included 48 children from the age group of 8 years to 14 years with a mean age of 10.12 years. The demographic characteristics of the study subjects are listed in Table 1.

The demographic characteristics showed that the mean age of the children enrolled in the study was $10.12 \pm 2.06$ years with the age range of 8-14 years which is commonly seen in Indian children of $6^{\text {th }}$ standard to $8^{\text {th }}$ standard which was the sample of the present trial. Concerning the education status, out of the total 48 subjects $39.58 \%(n=19)$ subjects were from standard $6^{\text {th }}, 37.5 \% \quad(n=18)$ subjects were from standard $7^{\text {th }}$, and 11 participants $(22.91 \%)$ were from $8^{\text {th }}$ standard. This shows uniform enrolment from class $6^{\text {th }}, 7^{\text {th }}$, and $8^{\text {th }}$. Regarding the socioeconomic status, it was seen that 14 subjects $(29.16 \%)$ were from the low socioeconomic background, the majority $54.16 \% \quad(n=26)$ were from the medium economic background, and least, i.e., $16.66 \%(\mathrm{n}=8)$ were from the high socioeconomic status.

The present trial also evaluated the factors and parameters as they relate to obesity in the study sample of 48 schoolgoing children. The results are depicted in Table 2. It was seen that 15 subjects were obese $(31.25 \%)$, 9 were overweight $(18.75 \%)$, and $50 \%$ of the study participants were healthy based on the BMI. Eating habits showed that majority subjects preferred junk from the school canteen which made $41.66 \% \quad(n=20), 22 \quad(4.83 \%)$ subjects got lunchbox from home, and 6 subjects $(12.5 \%)$ skipped lunch during school. $70.83 \%(\mathrm{n}=34)$ of subjects liked the physical activity during the school curriculum, whereas, $29.16 \%$ $(n=14)$ skipped the physical activity sessions of the school. Physical activity was avoided by $45.83 \%(n=22)$ subjects at home. Only $43.75 \% \quad(n=21)$ of subjects were seeing a specialized paediatrician for their treatments, $39.58 \%$ $(n=19)$ were visiting a general physician, and $16.66 \%(n=8)$ changed their primary health-care provider. For associated diseases, $4.16 \%(\mathrm{n}=2)$ subjects had diabetes, $22.91 \%(\mathrm{n}=11)$ participants had asthma, and $12.5 \%(\mathrm{n}=6)$ had adenoids. 
The study also assessed the counselling received by the study participant and the results are summarised in Table 3 . Counselling for nutrition, physical activity, and weight were provided to study participants and the results showed that $80 \%(\mathrm{n}=12)$ obese, $66.6 \%(\mathrm{n}=6)$ overweight, and $58.3 \%$ $(\mathrm{n}=14)$ healthy subjects received counselling for nutrition. $73.33 \%(n=11)$ obese, $655.5 \%(n=5)$ overweight, and no healthy subjects received counselling for physical activity. For weight, $66.6 \%(n=10)$ obese, $77.7 \%(n=7)$ overweight, and $54.16 \%(n=13)$ healthy subjects received counselling. In male participants $61.53 \% \quad(n=16), 69.23 \% \quad(n=18)$, and $65.38 \%(n=17)$ respectively were counselled for nutrition, physical activity, and weight. Similarly, in females $68.18 \%$ $(n=15), 63.63 \%(n=14)$, and $63.63 \%(n=14)$ respectively were counselled for nutrition, physical activity, and weight. Regarding diabetics in the study $(n=2)$, all the subjects were counselled for weight, nutrition, as well as physical activity. In subjects with asthma, the counselling for nutrition, physical activity, and weight was given to $63.6 \%(n=7)$,
$54.5 \%(n=6)$, and $54.5 \%(n=6)$ respectively.

Table 1: Demographic characteristics of the study participants

\begin{tabular}{|c|c|c|c|c|}
\hline S. No. & Characteristic & & $\begin{array}{c}\text { Number } \\
(\mathbf{n})\end{array}$ & $\begin{array}{c}\text { Percentage } \\
(\%)\end{array}$ \\
\hline 1. & Mean age & $10.12 \pm 2.06$ & & \\
\hline 2. & Age Range (in years) & $8-14$ years & & \\
\hline 3. & Gender & & & \\
\hline a. & Male & & 26 & $54.16 \%$ \\
\hline b. & Female & & 22 & $45.83 \%$ \\
\hline 4. & Education Status & & & \\
\hline a. & $6^{\text {th }}$ standard & & 19 & $39.58 \%$ \\
\hline b. & $7^{\text {th }}$ standard & & 18 & $37.5 \%$ \\
\hline c. & $8^{\text {th }}$ standard & & 11 & $22.91 \%$ \\
\hline 5. & $\begin{array}{c}\text { Socioeconomic } \\
\text { status }\end{array}$ & & & \\
\hline a. & Low & & 14 & $29.16 \%$ \\
\hline b. & Medium & & 26 & $54.16 \%$ \\
\hline c. & High & & 8 & $16.66 \%$ \\
\hline
\end{tabular}

Table 2: Characteristics of the study subjects as it relates to obesity

\begin{tabular}{|c|c|c|c|}
\hline S. No. & Characteristic & Number (n) & Percentage $(\%)$ \\
\hline 1. & \multicolumn{3}{|c|}{ BMI } \\
\hline a. & Obese (more than 95\%) & 15 & $31.25 \%$ \\
\hline b. & Overweight (less than/equal to $95 \%$ ) & 9 & $18.75 \%$ \\
\hline c. & Healthy $(<85 \%)$ & 24 & $50 \%$ \\
\hline 2. & \multicolumn{3}{|c|}{ Eating habits in school } \\
\hline a. & Lunchbox from home & 22 & $4.83 \%$ \\
\hline $\mathrm{b}$. & From Canteen & 20 & $41.66 \%$ \\
\hline c. & Skips Lunch & 6 & $12.5 \%$ \\
\hline 3. & \multicolumn{3}{|c|}{ Physical activity in school } \\
\hline a. & Joins & 34 & $70.83 \%$ \\
\hline b. & Skips & 14 & $29.16 \%$ \\
\hline 4. & \multicolumn{3}{|c|}{ Physical activity at home } \\
\hline a. & Yes & 26 & $54.16 \%$ \\
\hline b. & No & 22 & $45.83 \%$ \\
\hline 5. & \multicolumn{3}{|c|}{ Primary treatment centre } \\
\hline a. & Physician & 19 & $39.58 \%$ \\
\hline b. & Paediatrician & 21 & $43.75 \%$ \\
\hline c. & Variable & 8 & $16.66 \%$ \\
\hline 6. & \multicolumn{3}{|c|}{ Associated diseases } \\
\hline a. & Diabetes & 2 & $4.16 \%$ \\
\hline b. & Asthma & 11 & $22.91 \%$ \\
\hline c. & Adenoids & 6 & $12.5 \%$ \\
\hline
\end{tabular}

Table 3: Association of lifestyle modification with counselling and study parameters

\begin{tabular}{|c|c|c|c|c|}
\hline S. No. & Parameter & Counselling for nutrition & Counselling for physical activity & Counselling for weight \\
\hline 1. & \multicolumn{4}{|c|}{ BMI } \\
\hline a. & Obese (more than 95\%) & $80 \%(n=12)$ & $73.33 \%(n=11)$ & $66.6 \%(n=10)$ \\
\hline b. & Overweight (less than/equal to 95\%) & $66.6 \%(n=6)$ & $55.5 \%(\mathrm{n}=5)$ & $77.7 \%(\mathrm{n}=7)$ \\
\hline c. & \begin{tabular}{|c|} 
Healthy $(<85 \%)$ \\
\end{tabular} & $58.3 \%(\mathrm{n}=14)$ & - & $54.16 \%(\mathrm{n}=13)$ \\
\hline 2. & \multicolumn{4}{|c|}{ Eating habits in school } \\
\hline a. & Lunchbox from home & $50 \%(\mathrm{n}=11)$ & $22.72 \%(n=5)$ & $40.9 \%(n=9)$ \\
\hline b. & From Canteen & $70 \%(n=14)$ & $45 \%(n=9)$ & $65 \%(n=13)$ \\
\hline c. & Skips Lunch & $66.6 \%(n=4)$ & $83.3 \%(\mathrm{n}=5)$ & $33.3 \%(\mathrm{n}=2)$ \\
\hline 3. & \multicolumn{4}{|c|}{ Gender } \\
\hline a. & Male & $61.53 \%(\mathrm{n}=16)$ & $69.23 \%(n=18)$ & $65.38 \%(\mathrm{n}=17)$ \\
\hline b. & Female & $68.18 \%(\mathrm{n}=15)$ & $63.63 \%(\mathrm{n}=14)$ & $63.63 \%(n=14)$ \\
\hline 4. & \multicolumn{4}{|c|}{ Associated diseases } \\
\hline a. & Diabetes & $100 \%(\mathrm{n}=2)$ & $100 \%(n=2)$ & $100 \%(n=2)$ \\
\hline $\mathrm{b}$. & Asthma & $63.6 \%(n=7)$ & $54.5 \%(\mathrm{n}=6)$ & $72.72 \%(n=8)$ \\
\hline
\end{tabular}

\section{Discussion}

The present trial was carried out to evaluate the efficacy of implementing and identifying a novel strategy for preventing obesity in childhood. Also, the study was aimed at documenting the efficacy of counselling about physical activity, nutrition, and weight on obese children of low 
socioeconomic status. Approximately half of the study population of children in the present study were overweight and obese.

Although it is indicated that obese and overweight children should regularly and extensively receive counselling regarding nutrition, weight, and physical exercise, in the present study it was seen that counselling was not significantly different in obese children $(n=12,80 \%)$ compared to their healthy co-participants $(n=14,58.3 \%)$. These findings were in agreement with the findings by Taveras EM et al. ${ }^{[9]}$ in 2007 and Lazorick S et al. ${ }^{[10]}$ in 2011 where the author reported that healthy and obese both get similar counselling regarding their health. This difference could be because either the children are not attending the counselling sessions owing to underestimation of their weight or non-availability of counselling sessions in the school curriculum.

The present trial also evaluated the factors and parameters as they relate to obesity in the study sample of 48 schoolgoing children. It was seen that 15 subjects were obese $(31.25 \%)$, 9 were overweight $(18.75 \%)$, and $50 \%$ of the study participants were healthy based on the BMI. The present study calculated BMI to judge the obesity status where various other studies used height versus weight graph to judge overweight and obesity such as studies by Huang TT-K et al. ${ }^{[11]}$ in 2011 and Sesselberg TS et al. ${ }^{[12]}$ in 2010 where authors used height and weight graphs. However, the methods of our study to use BMI were consistent with the study by Keehbauch $\mathrm{J}$ et al. ${ }^{[13]}$ in 2012 where BMI was considered as a more reliable parameter of assessing obesity.

Eating habits showed that majority subjects preferred junk from the school canteen which made $41.66 \%(n=20), 22$ $(4.83 \%)$ subjects got lunchbox from home, and 6 subjects (12.5\%) skipped lunch during school. $70.83 \%(n=34)$ of subjects liked the physical activity during the school curriculum, whereas, $29.16 \%(n=14)$ skipped the physical activity sessions of the school. Physical activity was avoided by $45.83 \%(n=22)$ subjects at home. Only $43.75 \%(n=21)$ of subjects were seeing a specialized paediatrician for their treatments, $39.58 \% \quad(\mathrm{n}=19)$ were visiting a general physician, and $16.66 \%(\mathrm{n}=8)$ changed their primary healthcare provider. For associated diseases, $4.16 \%(n=2)$ subjects had diabetes, $22.91 \%(\mathrm{n}=11)$ participants had asthma, and $12.5 \%(n=6)$ had adenoids. These findings were consistent with the findings of O'Connor Duffany et al. ${ }^{[14]}$ in 2011 where similar results were reported.

Counselling for nutrition, physical activity, and weight were provided to study participants and the results showed that $80 \%(n=12)$ obese, $66.6 \%(n=6)$ overweight, and $58.3 \%$ $(\mathrm{n}=14)$ healthy subjects received counselling for nutrition. $73.33 \%(n=11)$ obese, $655.5 \%(n=5)$ overweight, and no healthy subjects received counselling for physical activity. For weight, $66.6 \%(n=10)$ obese, $77.7 \%(n=7)$ overweight, and $54.16 \%(\mathrm{n}=13)$ healthy subjects received counselling. In male participants $61.53 \% \quad(n=16), 69.23 \% \quad(n=18)$, and $65.38 \%(n=17)$ respectively were counselled for nutrition, physical activity, and weight. Similarly, in females $68.18 \%$ $(n=15), 63.63 \%(n=14)$, and $63.63 \%(n=14)$ respectively were counselled for nutrition, physical activity, and weight. Regarding diabetics in the study $(n=2)$, all the subjects were counselled for weight, nutrition, as well as physical activity. In subjects with asthma, the counselling for nutrition, physical activity, and weight was given to $63.6 \%(n=7)$,
$54.5 \%(\mathrm{n}=6)$, and $54.5 \%(\mathrm{n}=6)$ respectively. The results showed higher counselling received by girls than boys. This can be due to more concern regarding weight in girls than males. These findings were similar to the findings of Puhl RM et al. ${ }^{[15]}$ in 2007 and Tang Peronard JL et al. ${ }^{[16]}$ in 2008 where authors have reported similar results.

\section{Conclusion}

The present study concludes that overweight and obese children do not get more counselling sessions compared to their healthy peers. There is a need for more interactions at home and more reinforcement of counselling sessions in the school curriculum with special emphasis on overweight and obese children regarding their nutrition, physical activity, and weight. The present trial had few shortcomings including bias of geographical area; non-inclusion of the interaction of obese children to their parent/ teacher in study questionnaire, way of assessing the counselling availability was children response to the questionnaire which cannot be fully trusted, small sample size, and short monitoring period. The prospective longitudinal trials with a larger sample size and longer monitoring period are required to reach a definitive conclusion.

\section{References}

1. WHO. Consideration of the evidence on childhood obesity for the Commission on Ending Childhood Obesity: report of the Ad hoc Working Group on Science and Evidence for Ending Childhood Obesity, World Health Organization, Geneva 2016.

2. Lee YY, Kim M, Irfan M, Kim SH, Kim SD, Rhee MH. Physalis alkekengi Exhibits Antiobesity Effects in Mice with Potential of Inducing White Adipose Tissue Browning. J Med. Food 2020;23:312-8.

3. Hruby A, Hu FB. The epidemiology of obesity: a big picture. Pharmacoeconomics 2015;33:673-89.

4. Rankin J, Matthews L, Cobley S, Han A, Sanders R, Wiltshire HD et al. Psychological consequences of childhood obesity: psychiatric comorbidity and prevention. Adolesc Health Med Ther 2016;7:125-46.

5. Sashindran VK, Dudeja P. Obesity in School Children in India. Public Health in Developing Countries Challenges and Opportunities [Internet]. [Sashindran VK, Dudeja P. Obesity in School Children in India. Public Health in Developing Countries - Challenges and Opportunities 2020.

6. Pandita A, Sharma D, Pandita D, Pawar S, Tariq M, Kaul A. Childhood obesity: prevention is better than cure. Diabetes Metab Syndr Obes 2016;9:83-9.

7. Kelley CP, Sbrocco G, Sbrocco T. Behavioural Modification for the Management of Obesity. Primary Care-Clinics in Office Practice 2016, P159-75.

8. Kang JG, Park CY. Anti-obesity drugs: a review about their effects and safety. Diabetes Metab J 2012;36:1325.

9. Taveras EM, Sobol AM, Hannon C, Finkelstein D, Wiecha J, Gortmaker SL. Youths Perceptions of Overweight-related Prevention Counselling at a Primary Care Visit. Obesity 2007;15:831-6.

10. Lazorick S, Peaker B, Perrin EM et al. Prevention and treatment of childhood obesity: care received by a state medicaid population. Clin Pediatr (Phila) 2011;50:81626.

11. Huang TT-K, Borowski LA, Liu B et al. Pediatricians' 
and family physicians' weight-related care of children in the U.S. Am J Prev Med 2011;41:24-32.

12. Sesselberg TS, Klein JD, O’Connor KG, Johnson MS. Screening and counselling for childhood obesity: results from a national survey. J Am Board Fam Med 2010;23:334-42.

13. Keehbauch J, Miguel GS, Drapiza L, Pepe J, Bogue R, Smith-Dixon A. Increased documentation and management of paediatric obesity following the implementation of an EMR upgrade and education. Clin Pediatr (Phila) 2012;51:31-8.

14. O'Connor Duffany K, Finegood D, Matthews D et al. Community Interventions for Health $(\mathrm{CIH})$ : A novel approach to tackling the worldwide epidemic of chronic diseases. CVD Prevention and Control 2011;6:47-56.

15. Puhl RM, Latner JD. Stigma, obesity, and the health of the nation's children. Psychological Bulletin 2007; 133:557-80.

16. Tang-Péronard JL, Heitmann BL. Stigmatization of obese children and adolescents, the importance of gender. Obes Rev 2008;9:522-34. 